\title{
Attosecond pulses measured from the attosecond lighthouse
}

\author{
T. J. Hammond^, Graham G. Brown', Kyung Taec Kim²,3, D. M. Villeneuve ${ }^{1}$ and P. B. Corkum ${ }^{1}$
}

The attosecond lighthouse is a method of using ultrafast wavefront rotation with high-harmonic generation to create a series of coherent, spatially separated attosecond pulses. Previously, temporal measurements by photoelectron streaking characterized isolated attosecond pulses created by manipulating the single-atom response ${ }^{1-4}$. The attosecond lighthouse, in contrast, generates a series of pulses that spatially separate and become isolated by propagation. Here, we show that ultrafast wavefront rotation maintains the single-atom response (in terms of temporal character) of an isolated attosecond pulse over two octaves of bandwidth. Moreover, we exploit the unique property of the attosecond lighthouse-the generation of several isolated pulses-to measure the three most intense pulses. These pulses each have a unique spectrum and spectral phase.

When an intense ultrashort laser pulse (peak intensity $\sim 10^{14}-10^{15} \mathrm{~W} \mathrm{~cm}^{-2}$ ) is incident on a gas, coherent radiation spanning up to the extreme ultraviolet (XUV) or soft X-ray regimes can be created ${ }^{5}$. The radiation is emitted in a train of short, subcycle (of the driving field), attosecond ( 1 as $=10^{-18} \mathrm{~s}$ ) bursts ${ }^{6-10}$. If the driving field wavefront rotates at the focus on the timescale of an optical cycle, then each pulse is directed uniquely. It is predicted that this effect leads to a series of spatially separated, isolated attosecond pulses ${ }^{11}$. The pulses are such that the attosecond pulse train nature is still maintained in the nonlinear medium, and each sequential pulse is spatially separated in the far field, allowing for the study of both intra- and inter-pulse dynamics ${ }^{12}$.

Previously, the spatial separation of attosecond pulses has been demonstrated with spatially resolved spectra ${ }^{12,13}$. However, the formation of the isolated pulse at the far field has never been reported. We show that with an aperture in the far field, it is possible to isolate a single pulse in the series. We refocus the pulse to measure its temporal character. We also show that although the pulse selection process occurs in the far field, the single-atom response to the driving field in the generating medium determines the pulse characteristics. Finally, we present the photoelectron streaking measurements of three isolated pulses generated on subsequent half cycles, where the temporal character of the pulse is dependent on its time of creation within the driving field envelope.

In Fig. 1, we illustrate the generating medium response and the resulting pulse propagation to a strong driving field undergoing ultrafast wavefront rotation. The parameters used here match those of the experiment given in the Methods. We show the magnitude squared of the complex dipole response near the focus, calculated in the strong field approximation (SFA), as a function of time and the vertical displacement within the driving field. The rotating driving field generates a pulse train, where each pulse has a unique, rotated wavefront. The wavefront determines the direction of each pulse. The simulated pulses propagate with an angular divergence coordinate system, maintaining the temporal dependence of the pulse train and of each pulse.
In the far field, the pulses are now separated, and each pulse has a unique spectrum and spectral phase. The spatially resolved spectrum is projected on the right, and the bottom projection is the short-time Fourier transform showing that each pulse has a unique temporal character. Experimentally, we use an aperture in the far field to isolate a single pulse of interest, and refocus it to measure its temporal profile.

Spectral phase control is necessary in the generation of sub- 100 attosecond pulses, and will be crucial in reaching an atomic unit of time (24 as). Thus far, the spectral phase has been controlled by the anomalous group dispersion of filters ${ }^{14,15}$, pressure tuning of the generating medium ${ }^{16,17}$ and careful dispersion management in the $\mathrm{XUV}^{18,19}$. However, the spectral phase is also dependent on the driving field pulse envelope ${ }^{20}$. Judiciously selecting the attosecond pulse from the train may allow for improved pulse compression.

We select the most intense pulse, generated at the peak of the driving field, to measure the spectrum and spectral phase across the broadest energy range possible, and pass this pulse through a 200 -nm-thick beryllium filter. The generated spectrum spans the entire transmission bandwidth of nearly $90 \mathrm{eV}$. This bandwidth enables us to measure the spectral phase of an isolated attosecond pulse with photon energy spanning from just above the ionization potential $\left(I_{\mathrm{p}}\right)$ of neon through the plateau to the cutoff, a spectral breadth covering two octaves.

We reconstruct the isolated attosecond pulse in Fig. 2 using the principal component generalized projection algorithm (PCGPA) ${ }^{21,22}$, the details of which are provided in the Supplementary Information. In Fig. 2a, we show the measured spectrogram, which is taken over five optical cycles (one period $T_{\text {cycle }} \approx 2.53 \mathrm{fs}$ ) of the streaking field. The reconstructed spectrogram after $1 \times 10^{6}$ iterations is shown in Fig. 2 b.

The streaking-field free reference spectrum (orange) in Fig. $2 \mathrm{c}$ is compared with the reconstructed spectrum (blue). The most prominent difference is an amplitude modulation of the reconstructed spectrum due to the satellite pulses. The origin of the decreased modulation is due to the comparable spot sizes of the streaking field and the attosecond pulses, causing a spatially dependent streaking intensity ${ }^{23}$. The beryllium filter transmission bandwidth is shown in black for reference. The reconstructed pulse, shown in Fig. 2d has a full-width at half-maximum (FWHM) pulse duration of 310 as. The strong quadratic phase (green) implies a linear chirp responsible for the pulse duration being longer than its transform limit of 48 as. The inset is the logarithm of the intensity of the Fourier transform of the reference pulse (orange) showing the satellite pulse intensity $10^{-3}$ down from the main pulse, while the reconstructed pulse (blue) calculates the satellite pulse intensity to be $10^{-4}$ of the main pulse. Because we are taking the spatial average at the focus, we cannot detect any potential spatial chirp caused by the driving field wavefront rotation.

The strong quadratic phase is consistent with the high-harmonic generation process. In Fig. 3a, we calculate the single-atom dipole

\footnotetext{
Joint Attosecond Science Laboratory, University of Ottawa and National Research Council of Canada, 100 Sussex Drive, Ottawa K1N 6N5, Canada.

${ }^{2}$ Centre for Relativistic Laser Science, Institute for Basic Science (IBS), Gwangju 500-712, Republic of Korea. ${ }^{3}$ Department of Physics and Photon Science,

Gwangju Institute of Science and Technology (GIST), Gwangju 500-712, Republic of Korea. *e-mail: thammond@uottawa.ca
} 

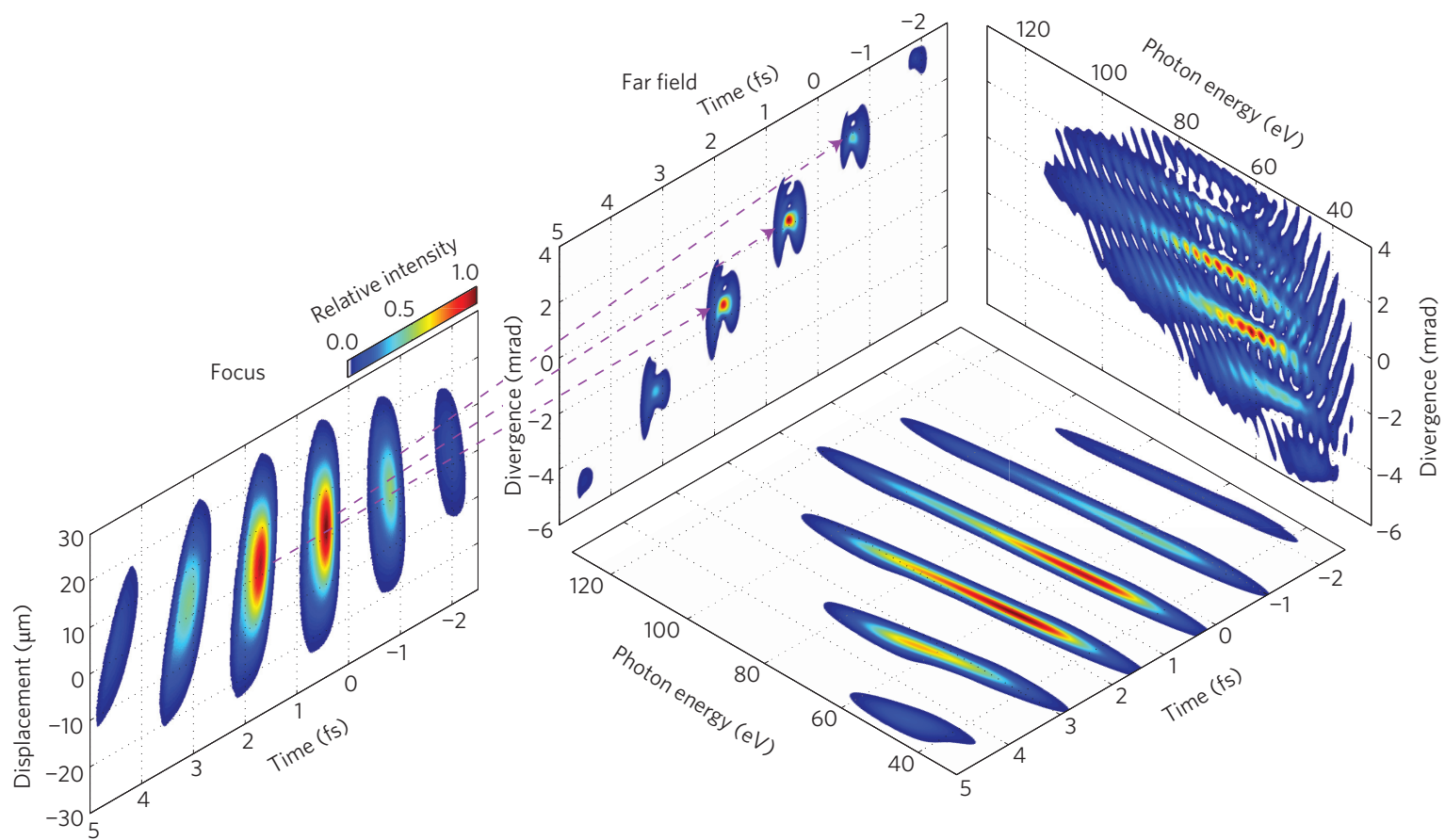

Figure 1 | Illustration of the attosecond lighthouse and the pulse-dependent spectral phase. Focus indicates the complex dipole intensity (short trajectory shown) response to a driving field undergoing ultrafast wavefront rotation, calculated in the strong field approximation. The three dashed lines represent the propagation of the pulses created at the rising, peak, and falling half-cycle of the driving field (top down, respectively). Far field: the attosecond pulses spatially separate; the right projection is the spatial-spectral image showing that each pulse has a unique spectrum, whereas the bottom projection is the short-time Fourier analysis illustrating that each pulse has a unique spectral phase. We use an aperture to isolate a single attosecond pulse, and we focus to reimage the generated pulse.
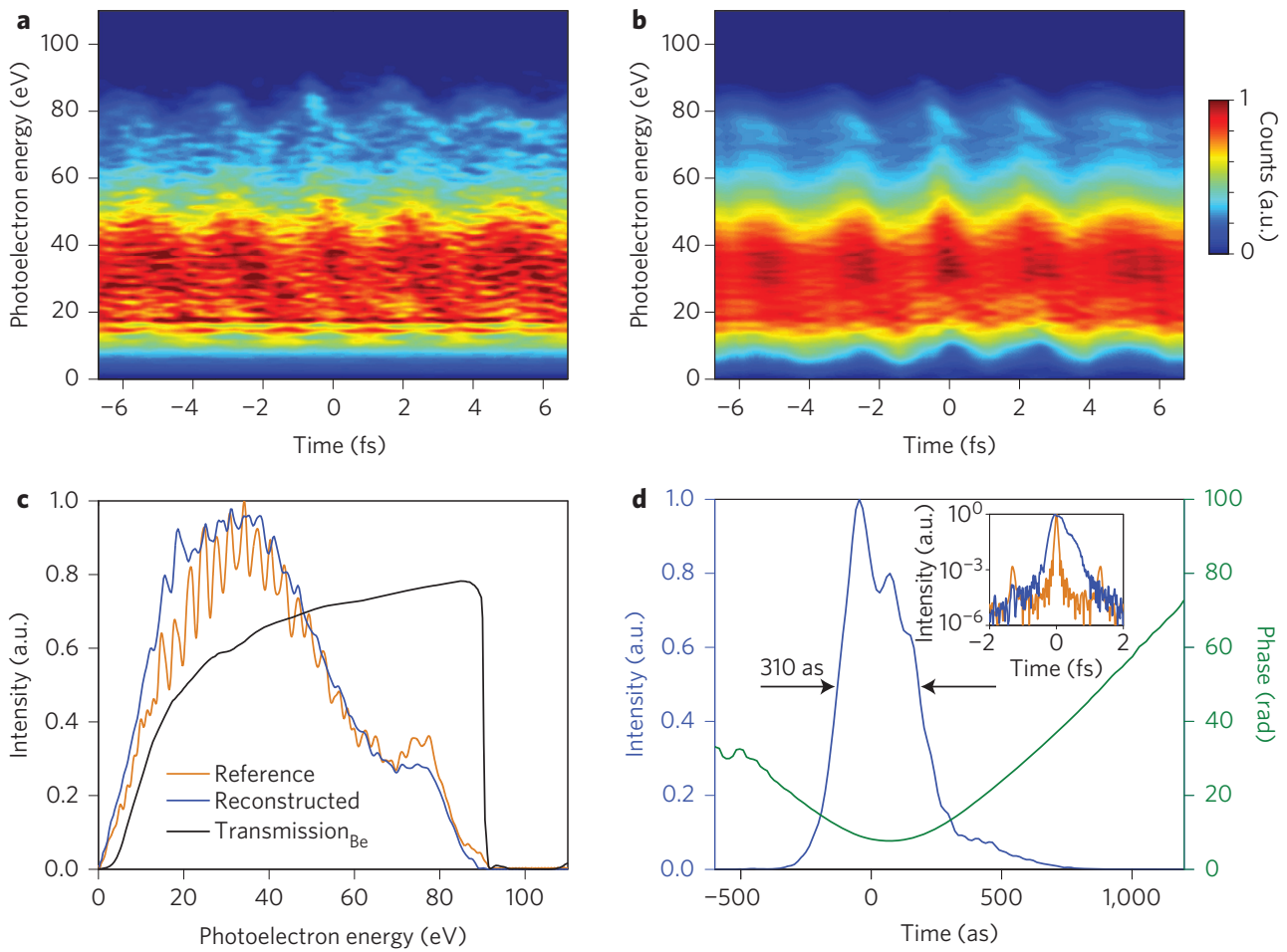

Figure $\mathbf{2}$ | Characterization of the pulse created at the peak of the driving field transmitted through a Be filter. a,b, Measured (a) and reconstructed (b) spectrograms. c, The attosecond pulse spectral intensity. The streaking-free spectrum is taken as a reference (orange). The reconstructed spectrum (blue) agrees well with the streaking-free spectrum although the spectral modulation amplitude is decreased. The Be filter transmission (black) nearly covers the entire generated spectrum. d, The reconstructed attosecond pulse (blue), with a measured 310 as FWHM pulse duration and its phase (green). The quadratic phase implies that the pulse is strongly chirped. Inset: log plot of the Fourier transform of the reference spectrum (orange) and the reconstructed pulse (blue). 

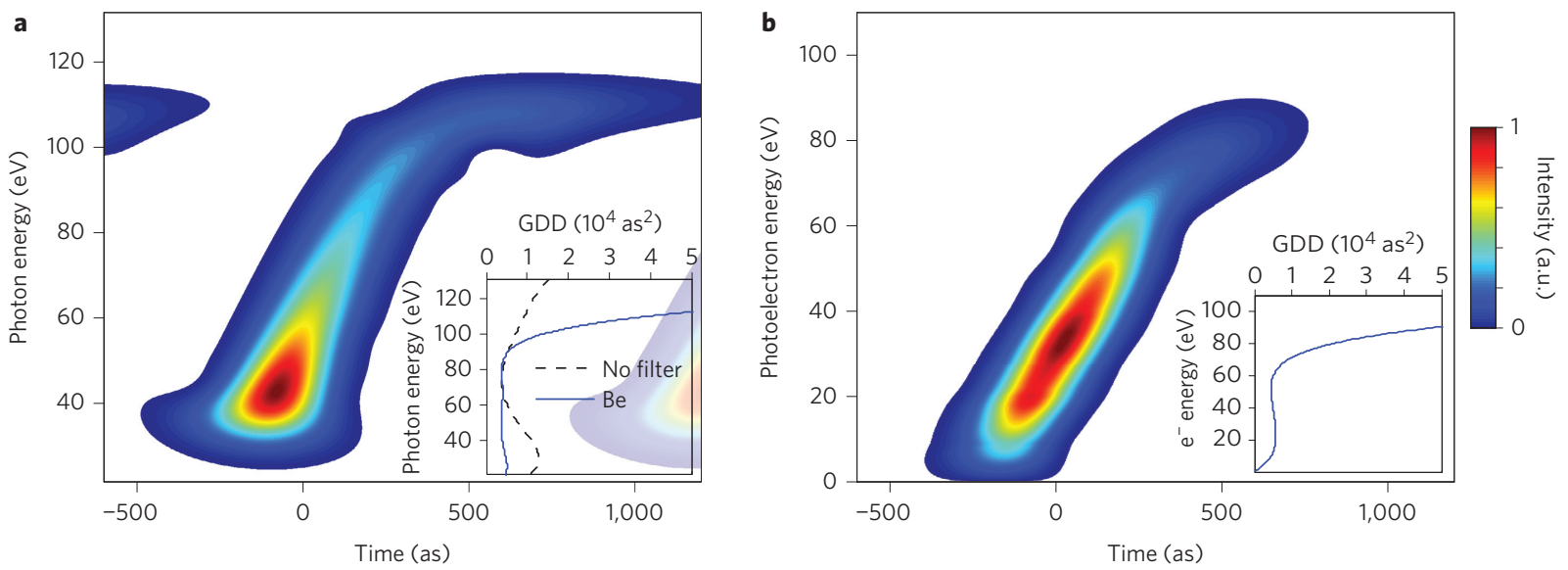

Figure 3 | Short-time Fourier transform of the pulse in Fig. 2. a, The SFA calculated single-atom response to the driving field. Inset: GDD of the dipole response without (black dashed) and including (blue) the Be filter dispersion. The anomalous dispersion of the Be filter compresses the pulse below $70 \mathrm{eV}$ (photon energy), while the Be K edge causes a large dispersion for photon energies $>100 \mathrm{eV}$. b. The reconstructed pulse. Inset: measured GDD. The good agreement of the reconstructed pulse with theory shows that the spectral phase of the attosecond pulse is not significantly distorted by the ultrafast wavefront rotation.
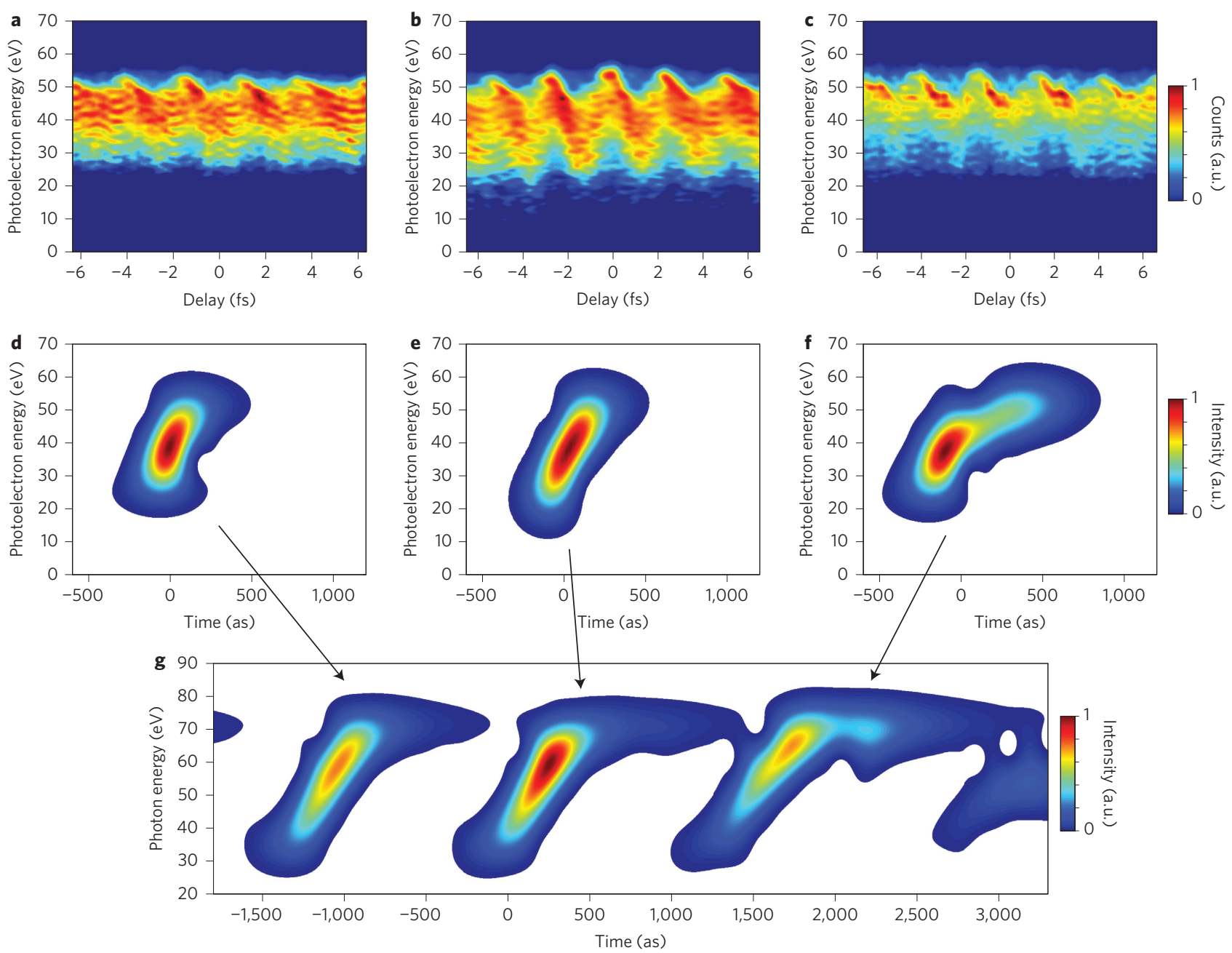

Figure 4 | Characterizing three isolated pulses transmitted through an Al filter. a-c, Measured spectrograms of the pulses created at the rising (a), peak (b), and falling (c) half cycle of the driving field using an Al filter. d-f, The short-time Fourier transform of the reconstructed pulses for the pulses generated at the rising (d), peak (e), and falling edge (f). $\mathbf{g}$, Short-time Fourier transform of the single-atom response to a generating field without wavefront rotation, but otherwise matching experimental conditions. Each pulse shows a unique spectrum and spectral phase. The time axes of panels $\mathbf{d}-\mathbf{f}, \mathbf{g}$ are scaled identically. The Al filter used to block the fundamental beam has an upper transmission cutoff at $51 \mathrm{eV}$ causing large dispersion above $\sim 45 \mathrm{eV}$ (photoelectron energy). 
response from a driving field not including wavefront rotation with characteristics matching the experiment (see Methods) using the strong-field approximation (SFA). The peak intensity is $5 \times 10^{14} \mathrm{~W} \mathrm{~cm}^{-2}$, with pulse duration of $6.25 \mathrm{fs}$ to include the increased pulse duration from spatial chirp, and carrier envelope phase (CEP) set to $-\pi / 4$. Using short-time Fourier analysis on the dipole response at the peak of the driving field (including the Be filter), the chirp is shown to be linear over most of the spectrum, spanning from 30 to $90 \mathrm{eV}$. Towards the cutoff region above $100 \mathrm{eV}$, there is a rapid change in the chirp due to the Be $\mathrm{K}$ edge. The inset shows the group delay dispersion (GDD) of the dipole response (black), and including the $200 \mathrm{~nm}$ thick Be filter (blue). The GDD is calculated using a seventh-order polynomial fit to the phase and taking its second derivative. The anomalous dispersion of the Be filter below $70 \mathrm{eV}$ results in a near-constant GDD from 30 to $90 \mathrm{eV}$. Above $100 \mathrm{eV}$, the GDD rapidly increases.

We compare the SFA result with Fig. 3b, the reconstructed pulse, which is a function of the photoelectron energy. The photoelectron energy is shifted down by $21.6 \mathrm{eV}$ (neon $I_{\mathrm{p}}$ ) relative to the photon energy. We see that again there are two regimes: below $70 \mathrm{eV}$ where there is a linear chirp, and above $70 \mathrm{eV}$ where the chirp rapidly increases. The inset shows the measured GDD, in good agreement with the SFA calculation. The agreement of the spectral phase between the SFA calculation of a single atom and the reconstructed pulse demonstrates that the temporal characteristics of the generated pulses are not significantly distorted by the wavefront rotation. Consequently, the attosecond lighthouse is a viable method to initiate or probe attosecond dynamics.

We are also able to measure the temporal characteristics of the pulses that precede and follow the pulse studied above. The spatially separated beams are independently and uniquely aligned with respect to the photoelectron spectrometer to produce their own spectrograms. For this part of the experiment, we use a $200 \mu \mathrm{m}$ thick aluminium filter to block the fundamental beam. The limited bandwidth of the Al filter allows for an increased count rate per energy bin, decreasing the acquisition time for each spectrogram. Due to the Al filter transmission up to $72 \mathrm{eV}$ (photon energy), the filter has a strong dispersion above $\sim 65 \mathrm{eV}$.

The measured spectrograms of the three largest amplitude pulses are shown in Fig. $4 \mathrm{a}-\mathrm{c}$, for the pulse generated on the rising edge, at the peak, and the falling edge of the generating field, respectively. The measured photoelectron bandwidths of the pulses are $\sim 25 \mathrm{eV}$ (transform limit $<100$ as), where the spectrum is determined largely by the $\mathrm{Al}$ filter. The temporal modulation of the spectrogram in $c$ is larger than the two preceding pulses, implying a more chirped pulse. Moreover, the amplitude of the satellite pulse in $c$ is significant, represented by the out-of-phase spectral shift, implying a larger content of the preceding pulse in its spectrogram.

In Fig. $4 \mathrm{~d}-\mathrm{f}$, we see the short-time Fourier transform of the reconstructed pulses, again generated in the rising edge, at the peak, and in the falling edge of the driving field, respectively. The reconstructed pulse generated in the falling edge in Fig. $4 \mathrm{f}$ shows a significantly increased chirp relative to the other two reconstructed pulses. We compare these reconstructed pulses with the SFA calculated single-atom dipole response (driving field parameters same as above), shown in Fig. 4g, including the effects of $200 \mathrm{~nm} \mathrm{Al}$ filter with an oxide layer. Here, the temporal character of each pulse is shown to be dependent on the time of creation within the driving field envelope. Although the measured spectrum is limited by the Al filter, the pulses show a significant difference in spectral phase, which is attributed to the generation process.

In conclusion, when high harmonics are generated by a field undergoing ultrafast wavefront rotation, each pulse in the train can be selected independently. We have used this feature to measure three beamlets. Each beamlet is an isolated attosecond pulse, and each has a unique spectral phase that arises from the sub-cycle time dependent field from which it was created. The measured characteristics are what we expect from the single-atom response: the wavefront rotation does not affect the attosecond pulse characteristics. This experiment not only confirms that a single beamlet can be selected and used, it also confirms an approach for creating the beams for attosecond pump-attosecond probe spectroscopy ${ }^{24-26}$.

\section{Methods}

Methods and any associated references are available in the online version of the paper.

Received 19 May 2015; accepted 11 December 2015; published online 18 January 2016

\section{References}

1. Sansone, G. et al. Isolated single-cycle attosecond pulses. Science 314, 443-446 (2006).

2. Goulielmakis, E. et al. Single-cycle nonlinear optics. Science 320, 1614-1617 (2008)

3. Feng, X. et al. Generation of isolated attosecond pulses with 20 to 28 femtosecond lasers. Phys. Rev. Lett. 103, 183901 (2009).

4. Ferrari, F. et al. High-energy isolated attosecond pulses generated by abovesaturation few-cycle fields. Nature Photon. 4, 875-879 (2010).

5. Brabec, T. \& Krausz, F. Intense few-cycle laser fields: frontiers of nonlinear optics. Rev. Mod. Phys. 72, 545-591 (2000).

6. Corkum, P. B. Plasma perspective on strong-field multiphoton ionization. Phys Rev. Lett. 71, 1994-1997 (1993).

7. Corkum, P. B., Brunett, N. H. \& Ivanov, M. Y. Subfemtosecond pulses. Opt. Lett. 19, 1870-1872 (1994)

8. Paul, P. M. et al. Observation of a train of attosecond pulses from high harmonic generation. Science 292, 1689-1692 (2001).

9. Mairesse, Y. et al. Attosecond synchronization of high-harmonic soft X-rays. Science 302, 1540-1543 (2003).

10. Krausz, F. \& Ivanov, M. Attosecond physics. Rev. Mod. Phys. 81, 163-234 (2009).

11. Vincenti, H. \& Quéré, F. Attosecond lighthouse: how to use spatiotemporally coupled light fields to generate isolated attosecond pulses. Phys. Rev. Lett. 108, 113904 (2012).

12. Kim, K. T. et al. Photonic streaking of attosecond pulse trains. Nature Photon. 7, 651-656 (2013)

13. Wheeler, J. A. et al. Attosecond lighthouses from plasma mirrors. Nature Photon. 6, 829-833 (2012)

14. Kim, K. T., Kim, C. M., Baik, M.-G., Umesh, G. \& Nam, C. H. Single sub-50attosecond pulse generation from chirp-compensated harmonic radiation using material dispersion. Phys. Rev. A 69, 051805 (2004).

15. López-Martens, R. et al. Amplitude and phase control of attosecond light pulses. Phys. Rev. Lett. 94, 033001 (2005).

16. Zhao, K. et al. Tailoring a 67 attosecond pulse through advantageous phasemismatch. Opt. Lett. 37, 3891-3893 (2012).

17. Ko, D. H., Kim, K. T. \& Nam, C. H. Attosecond-chirp compensation with material dispersion to produce near transform-limited attosecond pulses. J. Phys. B 45, 074015 (2012).

18. Hofstetter, M. et al. Attosecond dispersion control by extreme ultraviolet multilayer mirrors. Opt. Express 19, 1767-1776 (2011).

19. Bourassin-Bouchet, C. et al. Shaping of single-cycle sub-50-attosecond pulses with multilayer mirrors. New J. Phys. 14, 023040 (2012).

20. Naumov, A. Y., Villeneuve, D. M. \& Niikura, H. Contribution of multiple electron trajectories to high-harmonic generation in the few-cycle regime. Phys. Rev. A 91, 063421 (2015).

21. Kane, D. J. Recent progress toward real-time measurement of ultrashort laser pulses. IEEE J. Quant. Electron. 35, 421-431 (1999)

22. Mairesse, Y. \& Quéré, F. Frequency-resolved optical gating for complete reconstruction of attosecond bursts. Phys. Rev. A 71, 011401 (2005)

23. Chini, M., Wang, H., Khan, S. D., Chen, S. \& Chang, Z. Retrieval of satellite pulses of single isolated attosecond pulses. Appl. Phys. Lett. 94, 161112 (2009)

24. Goulielmakis, E. et al. Real-time observation of valence electron motion. Nature 466, 739-743 (2010)

25. Mauritsson, J. et al. Attosecond electron spectroscopy using a novel interferometric pump-probe technique. Phys. Rev. Lett. 105, 053001 (2010).

26. Lefebvre, C. et al. Attosecond pump-probe transition-state spectroscopy of laserinduced molecular dissociative ionization: Adiabatic versus nonadiabatic dressed-state dynamics. Phys. Rev. A 88, 053416 (2013). 


\section{Acknowledgements}

We gratefully acknowledge the technical assistance of D. Crane and B. Avery, and useful discussions with A. Naumov. We also acknowledge financial support from Canada's NSERC, NRC, CFI, and CRC, as well as from America's AFOSR and DARPA Pulse Program through a grant from AMRDEC.

\section{Author contributions}

T.J.H., K.T.K. and P.B.C. designed the experiment. T.J.H., G.G.B., and K.T.K. performed the experiment. K.T.K. and T.J.H. provided the theoretical analysis. T.J.H. analysed the experimental data. T.J.H. and P.B.C. prepared the initial manuscript. All authors contributed in writing the manuscript.

\section{Additional information}

Supplementary information is available in the online version of the paper. Reprints and permissions information is available online at www.nature.com/reprints. Correspondence and requests for materials should be addressed to T.J.H.

\section{Competing financial interests}

The authors declare no competing financial interests. 


\section{Methods}

The details of the laser are described elsewhere ${ }^{12}$. The pulse duration and carrier envelope phase (CEP) are finely controlled by a pair of parallel aligned wedges. The wavefront rotation is created by a pair of misaligned wedges, with the second wedge rotated by $25^{\circ}$ from normal. The pulse duration is increased by the wavefront rotation to an estimated $6.25 \mathrm{fs}$, while the spot size at the focus is measured to be $50 \times 35 \mu \mathrm{m}^{2}$, leading to an estimated peak intensity of $5 \times 10^{14} \mathrm{~W} \mathrm{~cm}^{2}$. For the streaking arm, $25 \mu \mathrm{J}$ is separated by a broadband beamsplitter and delayed using a computer controlled delay stage, with 150 as steps. The streaking field intensity is estimated to be $2 \times 10^{12} \mathrm{~W} \mathrm{~cm}$.

To ensure that we are characterizing the pulse of interest, a $200-\mu \mathrm{m}$-thick $\beta$-barium borate (BBO) crystal is used to generate $3 \mu \mathrm{J}$ of the second harmonic of the driving field ${ }^{27}$. A 1 -mm-thick calcite plate and $\lambda / 2$ waveplate are used to tune the phase and align the polarization of the fields, where the relative intensity at the focus is $I_{2 \omega} / I_{\omega} \sim 3 \times 10^{-3}$. By tuning the phase of the second harmonic, we are able to enhance the desired beamlet while suppressing the preceding and following pulses. We align the generating arm such that the pulse of interest passes through an aperture ( $2 \mathrm{~mm}$ diameter); the attosecond pulse dictates the propagation axis. We optimize the count rate from the photoelectron spectrometer (PES) and compare its spectrum with the spatially resolved spectrum. We use this information to align the desired pulse to the photoelectron spectrometer, and then misalign the $\mathrm{BBO}$ to remove the second harmonic when recording the spectra.

Further details of the experimental set-up can be found in the Supplementary Information.

\section{References}

27. Hammond, T. J., Kim, K. T., Zhang, C., Villeneuve, D. M. \& Corkum, P. B. Controlling attosecond angular streaking with second harmonic radiation. Opt. Lett. 40, 1768-1770 (2015). 


\title{
Attosecond pulses measured from the attosecond lighthouse
}

\author{
TJ Hammond, ${ }^{1, *}$ Graham G. Brown ${ }^{1}$, Kyung Taec Kim ${ }^{2,3}$, \\ D. M. Villeneuve ${ }^{1}$, and P. B. Corkum ${ }^{1}$ \\ ${ }^{1}$ Joint Attosecond Science Laboratory, \\ University of Ottawa and National Research Council of Canada, \\ 100 Sussex Dr, Ottawa, Canada, K1N 6N5, \\ ${ }^{2}$ Centre for Relativistic Laser Science, Institute for Basic Science (IBS), Korea \\ ${ }^{3}$ Department of Physics and Photon Science, GIST, Korea
}

\section{Experimental Setup and Methods}

The experimental setup is shown in Fig. S1. We use a $1 \mathrm{kHz}, 1.5 \mathrm{~mJ}, 25$ fs Ti:Sapphire amplifier with central wavelength of $800 \mathrm{~nm}$. These pulses are focussed into a differentially pumped neon filled hollow core fiber with $250 \mu \mathrm{m}$ inner diameter at 1.25 Bar, followed by compression using a series of reflections from chirped mirrors. This setup generates the spectrum necessary for sub-5 fs pulses centered at $760 \mathrm{~nm}$. The pulse duration and carrier envelope phase (CEP) are finely controlled by a pair of parallel aligned wedges (W1). The wavefront rotation is created by a pair of misaligned wedges (W2), with the second wedge rotated by $25^{\circ}$ from normal. For the streaking arm, $25 \mu \mathrm{J}$ is separated by a broadband beamsplitter (BS) and delayed using a computer controlled delay stage (DS), with 150 as steps. The streaking field intensity is estimated to be $2 \times 10^{12} \mathrm{~W} / \mathrm{cm}^{2}$. 


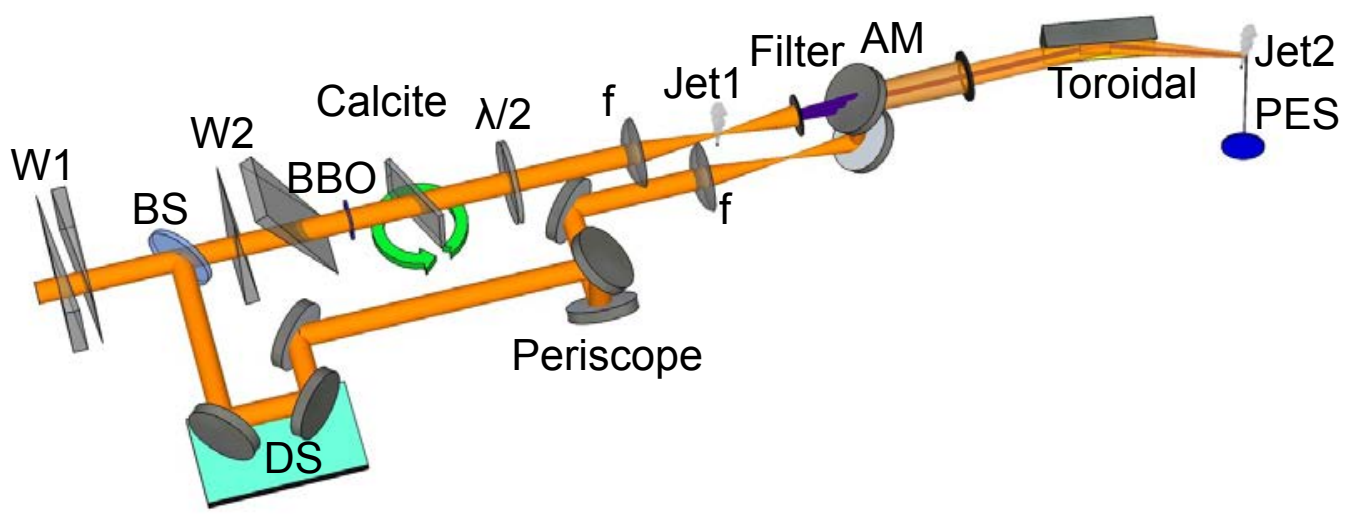

Figure S1: Experimental setup; see text for details. The generation arm directs the attosecond pulse into the aperture (AM), which isolates the pulse. The streaking arm has matching focussing optics such that the streaking field and the attosecond pulse foci overlap in the detection gas (Jet2) of the photoelectron spectrometer (PES).

The generating field, now with $200 \mu \mathrm{J}$ energy pulses, is passed into a vacuum chamber and is focussed onto a neon jet (Jet1; backing pressure 4 Bar, diameter $250 \mu \mathrm{m}$, placed $1 \mathrm{~mm}$ before focus) by a $300 \mathrm{~mm}$ focal length mirror (f), shown as a lens for simplicity. The spot size is $35 \mu \mathrm{m}$ by $50 \mu \mathrm{m}$ and the estimated full width at half maximum pulse duration is increased from the initially 4.5 fs (measured by a home-built Spectral Phase Interferometry for Direct Electric-field Reconstruction - SPIDER) to an estimated 6.25 fs, with an estimated peak intensity of $(5 \pm 0.5) \times 10^{14} \mathrm{~W} / \mathrm{cm}^{2}$, attaining a maximum measured photon energy of $115 \mathrm{eV}$.

The filters, either a $200 \mathrm{~nm}$ thick Be or a $200 \mathrm{~nm}$ thick $\mathrm{Al}$, separate the generated pulses from the driving field. The generated and streaking fields are recombined using an aperture mirror (AM), $2 \mathrm{~mm}$ diameter aperture, and focussed using a gold-coated toroidal mirror at $5^{\circ}$ grazing incidence, $380 \mathrm{~mm}$ focal length.

The time-of-flight PES uses neon as the detecting gas, separated from the detector by $0.5 \mathrm{~m}$ magnetic shielded tube. Einzel lenses are used to increase the count rate. For 
the measurements with the Be filter, we apply $13 \mathrm{~V}$ to increase the count rate of the low energy electrons (spanning $0-20 \mathrm{eV}$ ). For the measurements with the Al filter, we apply $40 \mathrm{~V}$ to increase the electron count rate over the energy range $30-50 \mathrm{eV}$. We calibrate the photoelectron spectrum by a reference spectrum with each filter, without the einzel lens applied and without the streaking field, over $1.2 \times 10^{5}$ laser shots. The total counts for each step of the spectrogram is approximately 9000 for Be, and 4500 for Al. The photoelectron spectrum minimum bin resolution is 0.1 ns for an estimated resolution of greater than $0.1 \mathrm{eV}$ at a photoelectron energy of $50 \mathrm{eV}(1)$.

\section{Reconstruction of the Gate}

The spectrograms shown in the main text, Figs. 2 and 4, do not cover sufficient time delay to fully reconstruct the streaking field, i.e. the gate. Here, in Fig. S2(a), we show the spectrogram of the largest amplitude pulse with the Al filter over a 35 fs window. The much larger delay requires a larger interpolated matrix $(2048 \times 2048)$ to satisfy the Nyqvist sampling criterion. Using the Principal Component Generalized Projection Algorithm (PCGPA), we reconstruct the spectrogram, shown in (b). The short-time Fourier transform of the reconstructed attosecond pulse, shown in (c), is nearly identical to the reconstructed pulse shown in Fig. 4(e) of the main text. Because of the longer time delay, we can now reconstruct the streaking field, shown in (d). The reconstructed field (red) is fit using a non-linear regression algorithm to give a pulse duration of $7.4 \mathrm{fs}$ and a central wavelength of $770 \mathrm{~nm}$.

The increased pulse duration of the streaking field relative to the generating field is due to residual dispersion. The measured generating field pulse duration is $4.5 \mathrm{fs}$ from a SPIDER - Spectral Phase Interferometry for Direct Electric-field Reconstruction, and the measured central wavelength is $760 \mathrm{~nm}$. The resulting pulse duration caused by the 


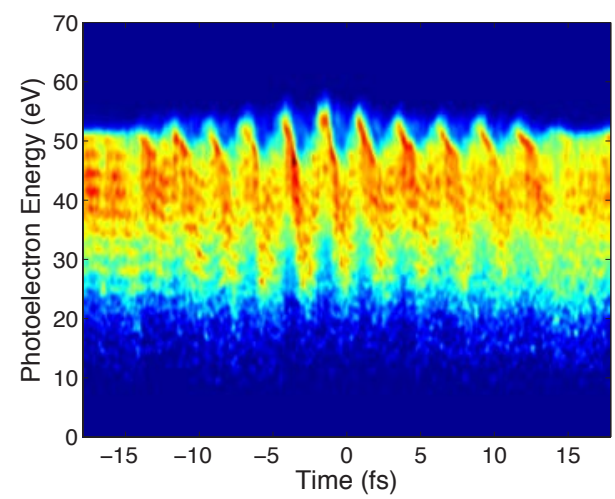

(a)

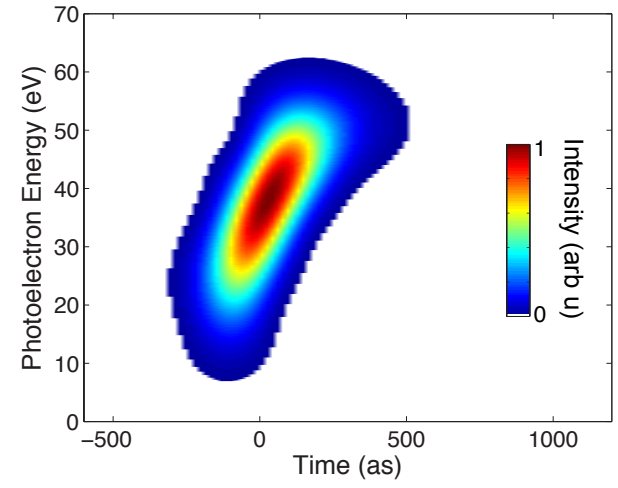

(c)

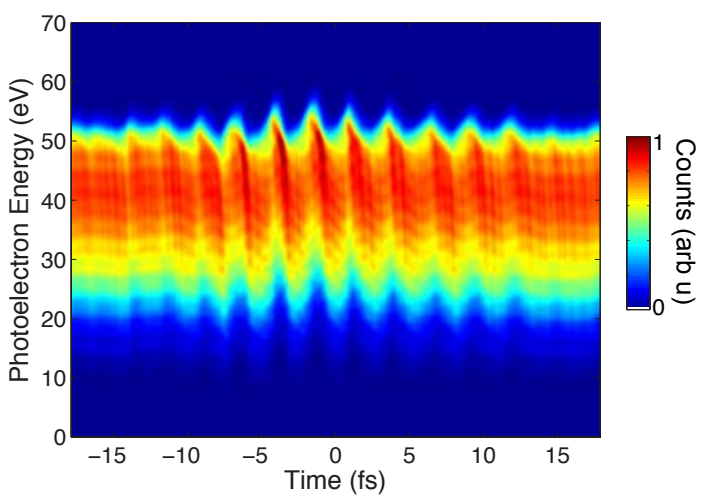

(b)

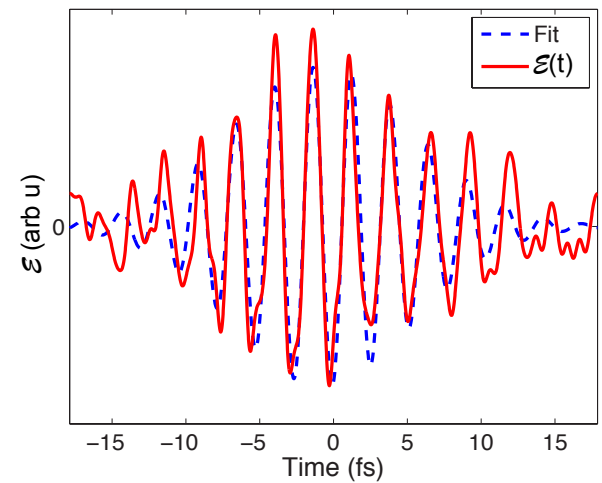

(d)

Figure S2: Reconstruction of the pulse created at the peak of the driving field, transmitted through the Al filter. (a) The spectrogram spanning 35 fs; (b) the reconstructed spectrogram using PCGPA; (c) short-time Fourier transform of the reconstructed attosecond pulse, and (d) the reconstructed streaking electric field. The reconstructed streaking field (red) is fit to a pulse duration of $7.4 \mathrm{fs}$ with a central wavelength of $770 \mathrm{~nm}$ (blue dash). 
group delay dispersion (GDD) imposed on a transform limited pulse of such bandwidth and central wavelength passing through $0.24 \mathrm{~mm}$ of fused silica is $7.4 \mathrm{fs}$.

The reason for the non-transform limited streaking field is the following. The beam is split into the generating arm and the streaking arm before the introduction of the misaligned BK7 wedges (see the Methods section). After tilting the wedges in the generating arm to find the optimum wavefront rotation to maximize the beamlet separation, we introduce fused silica glass to the streaking arm. We assume that the generating arm is transform limited, and we spectrally interfere the generating arm with the streaking arm after the photoelectron spectrometer to minimize the difference in dispersion. However, due to the induced spatial chirp, the spectrum of the generating arm (now reduced in bandwidth) is not identical to that of the streaking arm (nearly maintaining the original spectrum). The modulation in the spectrum that we use to determine the relative dispersion is therefore not uniform over the entire streaking bandwidth. In order to fully compress the streaking field, a series of spectrograms are necessary, testing the dispersion of the streaking arm each time.

\section{Simulated Pulse}

The isolation of the attosecond pulses created by ultrafast wavefront rotation is achieved in the far field with an aperture. To predict the pulse contrast, we investigate the dipole response at the focus and the far field propagation of the attosecond pulses. For the theoretical calculations, we match the experimental conditions with an initially transform limited pulse of 4.5 fs pulse duration (full-width-at-half-maximum) and spot size of $35 \mu \mathrm{m}$ and central wavelength of $760 \mathrm{~nm}$. Due to the wavefront rotation, the initially few-cycle pulse is increased in pulse duration and spot size (in the rotating wavefront plane) (2), each by $\sqrt{2}$. The dipole response is calculated $1 \mathrm{~mm}$ before the focus to minimize the 
divergence of the created pulses. The estimated experimental peak intensity is $5 \times 10^{14}$ $\mathrm{W} / \mathrm{cm}^{2}$.

In Fig. S3, we compare the time dependent dipole response created near the focus and after propagation when generated by ultrafast wavefront rotation. The dipole response is calculated by the strong field approximation (SFA) (3) using non-adiabatic tunnel ionization (4), and with the long trajectory artificially removed. Near the focus, we see a train of attosecond pulses created because there is no temporal gate present, shown in Fig. S3(a). The short-time Fourier transform of the on-axis dipole moment is shown in Fig. S3(c).

The far field case uses propagation similar to Ref. (5). The wavefront rotation creates a series of attosecond pulses with unique propagation directions. In the far field, a 2 $\mathrm{mm}$ aperture is placed $1 \mathrm{~m}$ from the focus to isolate a single pulse, represented by the dark box in Fig. S3(b). The carrier envelope phase (CEP) of the driving field is set so that the pulse created at the peak of the field passes through the aperture, at $-\pi / 4$. We do not take the dispersion of the refocussing mirror (experimentally a gold toroidal mirror at $5^{\circ}$ grazing) into account. The short-time Fourier transform of the isolated pulse (intensity is log scaled) is shown in Fig. S3(d). Although the pulse is now isolated with the amplitude of the leading and tailing satellite pulses containing approximately $1 \%$ of the total intensity, the overlapping spectral portion is not uniform.

The far field spectrum of the pulse passing through the aperture, shown in Fig. S4(a), has strong modulation for lower energies due to the divergence of the satellite pulses. To optimize the isolation of the beamlets created by the short trajectory, the nonlinear medium is placed before the focus using the converging wavefront of the driving field to compensate for the diverging wavefront of the generated field. However, this compensation occurs over a limited portion of the spectrum and the result is a spectrally dependent 


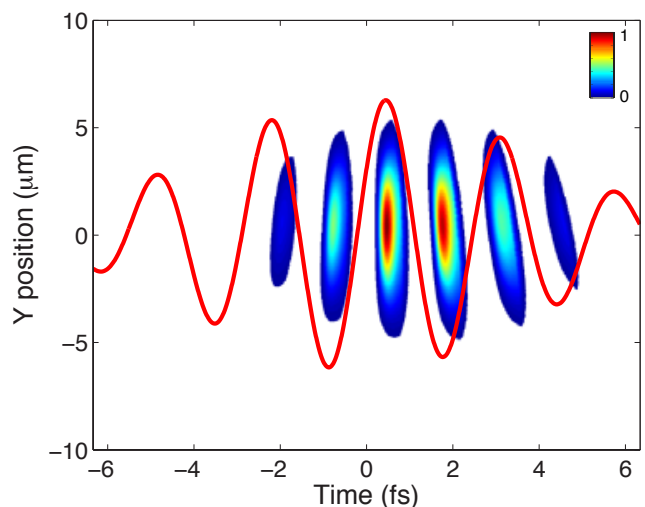

(a)

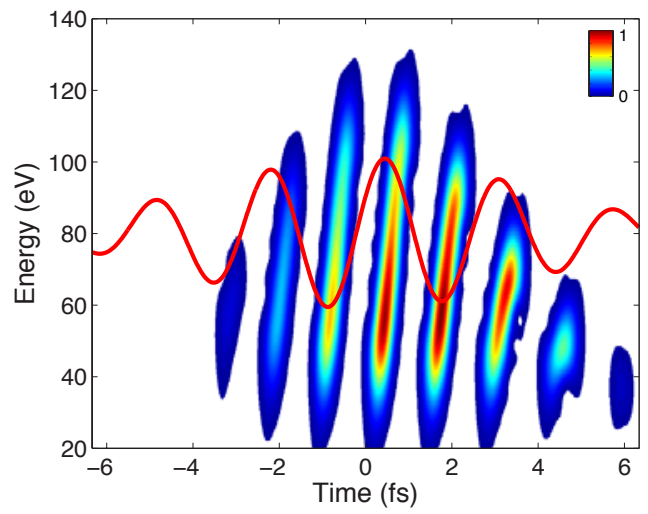

(c)

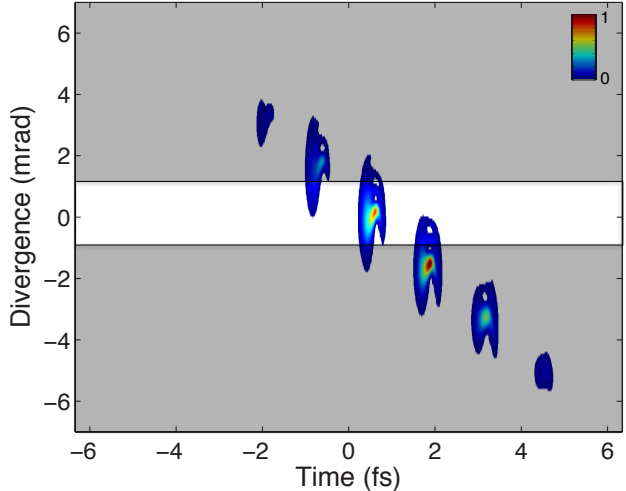

(b)

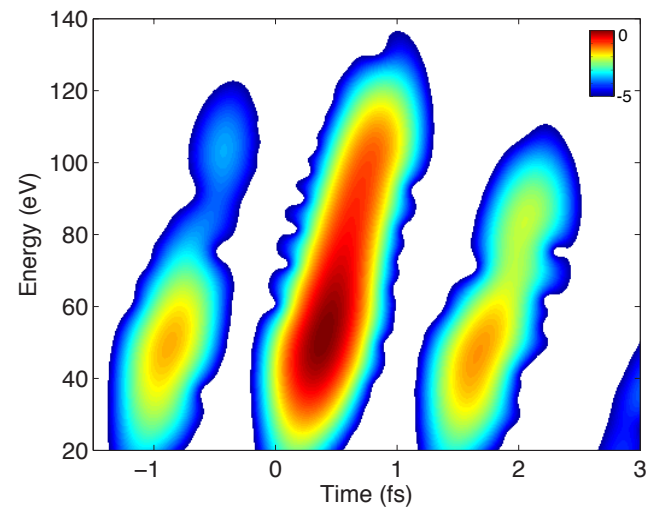

(d)

Figure S3: (a) Simulated dipole intensity $1 \mathrm{~mm}$ before the focus, matching experimental conditions, central wavelength of driving field shown; (b) the resulting far field spatial separation of the attosecond pulse train, where the greyed area represents the blocked beamlets by the $2 \mathrm{~mm}$ diameter aperture $1 \mathrm{~m}$ from the focus; (c) short-time Fourier transform of the on-axis dipole moment, and (d) amplitude of the short-time Fourier transform of the transmitted beamlet. The portion of the satellite beamlets that is transmitted is spectrally dependent. Colour code: relative intensity in (a)-(c) linear, (d) logarithmic scales. 
pulse contrast. Here, we include the transmission of the $200 \mu \mathrm{m}$ Be filter (black solid) to compare the simulated spectrum with experiment (Fig 2(d) of the main text). We also show the effect of the $200 \mathrm{~nm}$ thick Al filter (black dashed) with $15 \mathrm{~nm}$ thick oxide layer total (black dotted) (6) on the spectrum. The pulse duration also changes due to the filters, as shown in Fig. S4(b). The Be filter compresses the low energy portion of the spectrum (green), while strongly distorting the higher energy. Additionally shown is the effect of the Al filter (red), where the spectrum is limited due the Al L2 edge, increasing the pulse duration. The phase of the pulses transmitted is shown in Fig. S4(c). The phase of the unfiltered pulse (blue) is nearly quadratic over the main portion of the pulse, implying a linear chirp. The anomalous dispersion of the Be filter results in a flatter phase at the beginning of the pulse, compressing the pulse slightly.

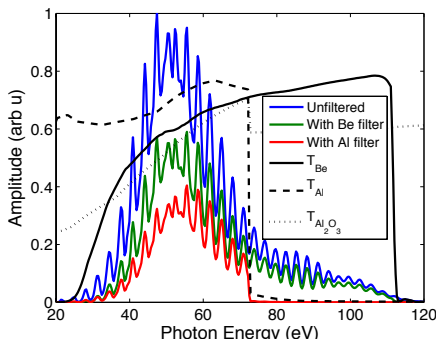

(a)

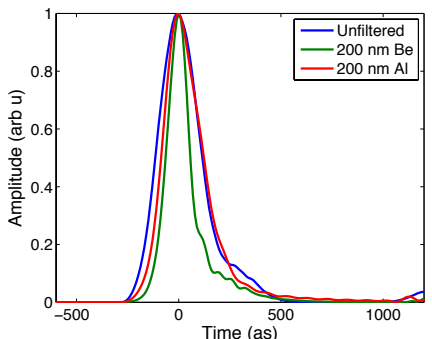

(b)

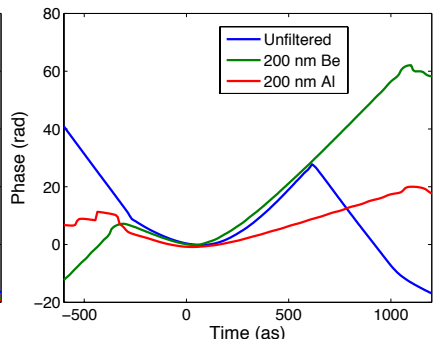

(c)

Figure S4: (a) The calculated spectrum of the pulse passing through a $2 \mathrm{~mm}$ aperture $1 \mathrm{~m}$ from the focus. The resulting spectrum without a filter (blue), with $200 \mathrm{~nm}$ thick Be filter (green), and with $200 \mathrm{~nm}$ thick Al filter with oxide layer (red). The transmission of the Be filter (black solid) transmits nearly the entire spectrum, while the Al filter truncates much of the higher portion (black dashed). The Al oxide layer is assumed to be $15 \mathrm{~nm}$ thick (total), attenuating the lower portion of the spectrum. The resulting transmitted pulse intensity (b) and phase (c) without a filter (blue), and through the Be (green) or $\mathrm{Al}$ (red) filters. 


\subsection{Effect of Weak Satellite Pulses on Streaking}

The effect of the satellite pulses on the spectrogram is shown in Fig. S5. The spectrograms are created by matching the experimental conditions of the attosecond lighthouse, propagating the generated pulses $1 \mathrm{~m}$, pass through the filter and a $2 \mathrm{~mm}$ diameter aperture, and refocussing to the second detection chamber. The detection gas has $I_{p}=21.6$ $\mathrm{eV}$, and the streaking field pulse duration is $7 \mathrm{fs}$ with a peak intensity of $2 \times 10^{12} \mathrm{~W} / \mathrm{cm}^{2}$. The different metal filters lead to different bandwidths in the spectrograms, with (a) 200 nm Be filter, and (b) $200 \mathrm{~nm} \mathrm{Al}$.

The effect of the satellite pulses on the spectrogram with the Be filter has three regimes. In the low photoelectron energy regime, where the two satellite pulses have approximately $1 \%$ of the total intensity, the spectrogram has a RABBIT-like (resolution of attosecond beating by interference of two-photon transitions) structure due to the pulse train ( 7$)$. In higher photoelectron energy, the satellite pulses are weaker. Here, the spectral modulation follows the streaking field. In the third, highest, energy regime, the Be $\mathrm{K}$ edge leads to significant dispersion, where the spectrogram is distorted.

The Al filter shows similar characteristics, but the L2 absorption edge occurs where the spectral amplitude is strong, around $51 \mathrm{eV}$ in photoelectron energy. The two satellite pulses contribute to the momentum shift in the spectrogram opposite to the pulse of interest. The effect of the satellite pulses along with the dispersion of the Al L2 absorption edge makes differentiating their contributions difficult.

We can compare the spectral modulation due to the satellite pulses with and without the streaking field. In both the experiment and simulation, we see a $20 \%$ modulation in the spectrum without the streaking field at the peak of the spectrum, indicating a $1 \%$ satellite pulse amplitude. However, in the experiment (Figs 2(a) and 4(b) of the main text), the spectrograms show a decreased amplitude of the spectral modulation, resulting 
in a decreased reconstructed satellite pulse amplitude relative to the simulation.

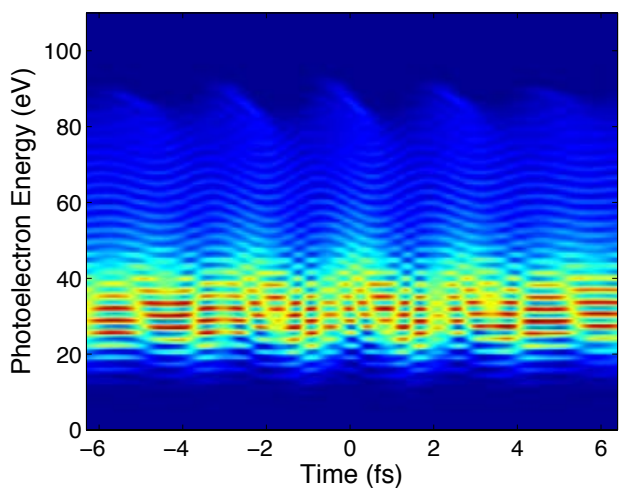

(a)

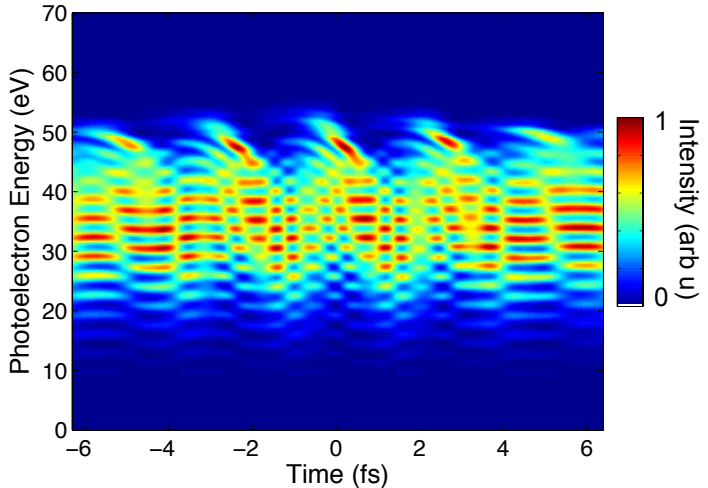

(b)

Figure S5: The calculated spectrograms based on experimental parameters with the Be filter (a) and Al filter (b). The satellite pulses cause a spectral modulation. In the low energy portion, the interference of the three pulses causes a beating, while in the higher portion of the spectrum the spectral modulation follows the streaking field. Both filters have absorption edges that have significant dispersion that distort the spectrograms.

\subsection{Principal Component Generalized Projection Algorithm}

The simulated pulses are reconstructed using the Principal Component Generalized Projection Algorithm (PCGPA). It is known that the PCGPA is limited due to the central momentum approximation (8) and the high streaking field strength required to characterise large bandwidth pulses. Here, we test the robustness of the PCGPA in reconstructing attosecond pulses.

To confirm that the streaking field strength and attosecond pulse bandwidth are appropriate for being reconstructed by the PCGPA, we have simulated the experimental conditions. For the Al filter, the algorithm quickly converged due to the limited bandwidth. However, for the Be filter, we found that using the full-width-at-half-maximum (FWHM) of the reconstructed pulse duration for measuring the convergence of the PCGPA is a bet- 
ter metric than the error because the error quickly converges whereas the FWHM does not. With the Be filter, the algorithm needs $>10^{5}$ iterations (in both experimental data and simulations) to converge.

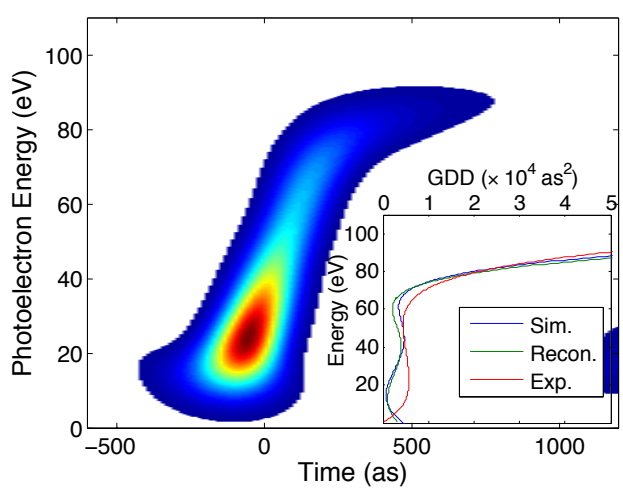

(a)

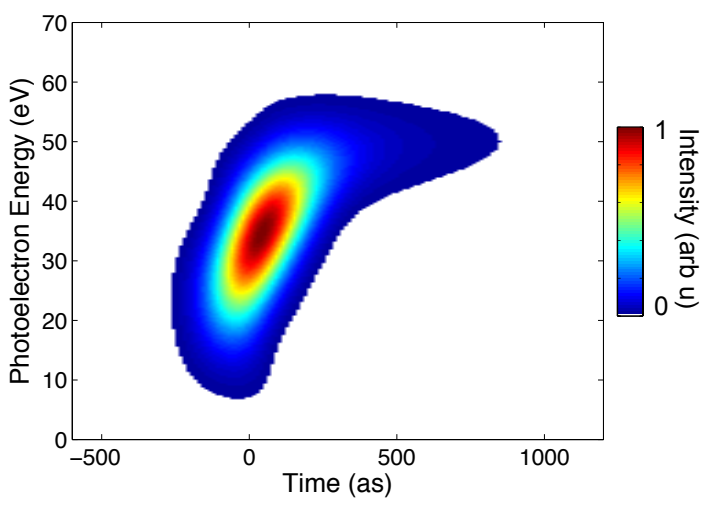

(b)

Figure S6: Reconstructed attosecond pulse from photoelectron streaking simulation; included are the generation, propagation, and filter. (a) The short-time Fourier analysis of the reconstructed pulse with the Be filter is in good agreement with the simulated pulse. Inset: calculated GDD of the simulated pulse (blue, photon energy down shifted by $I_{p}=21.6 \mathrm{eV}$ ), reconstructed pulse using PCGPA (green), and the experimental reconstructed pulse (red). (b) The short-time Fourier transform of the reconstructed simulated pulse including the Al filter.

The reconstructed pulses from the simulated photoelectron spectrograms are shown in Fig. S6. The effect of the Be filter on the attosecond pulse is shown in (a). Again, the chirp is shown to be linear over most of the spectrum, and near the Be K edge shows a significant increase in dispersion. The inset is the group delay dispersion (GDD) for the simulated attosecond pulse (blue) with the photon energy shifted by $I_{p}=21.6 \mathrm{eV}$ to account for the ionization potential of the detecting gas. The GDD of the reconstructed simulated pulse (green) agrees well, demonstrating that the PCGPA algorithm can accurately reconstruct the attosecond pulse over such a broad spectrum. The GDD of the 
experimentally reconstructed attosecond pulse (red) agrees with the simulation. In the case of the Al filter (b), the filter transmission limits the bandwidth and increases the dispersion near the absorption edge.

\section{Comparison with PROOF}

Reconstruction of the spectral phase by Phase Retrieval by Omega Oscillation Filtering (PROOF) is also performed on the experimental data. This method has been shown to accurately characterize ultrabroadband and ultrashort pulses $(9,10)$ without the approximations required by PCGPA. To calculate the spectral phase in PROOF, we use an iterative algorithm that calculates the dispersion coefficients up to the $9^{\text {th }}$ order.

The reconstructed phase by PROOF and PCGPA is shown in Fig. S7(a), along with the calculated dispersion in Fig. S7(b). In the largest amplitude portion of the spectrum, the phase (and dispersion) from PCGPA and PROOF match well. However, in spite of the high polynomial order, we found that PROOF did not match the large dispersion from the Be K edge. This is likely due to the optimization of the fit, which weighs the spectral amplitude. Over the largest amplitude portion of the spectrum, the dispersion is approximately $4500 \mathrm{as}^{2}$ calculated by both algorithms, and agrees well with simulation. The short time Fourier transform of the reconstructed pulse by PROOF is shown in Fig. $\mathrm{S} 7(\mathrm{c})$. 


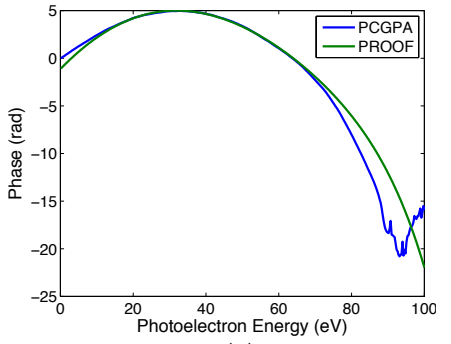

(a)

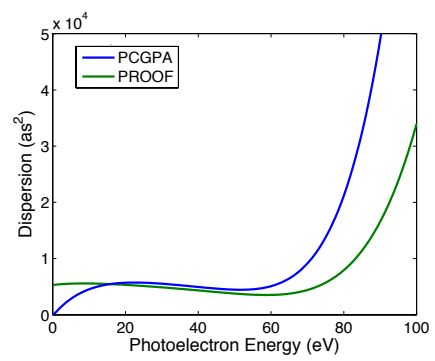

(b)

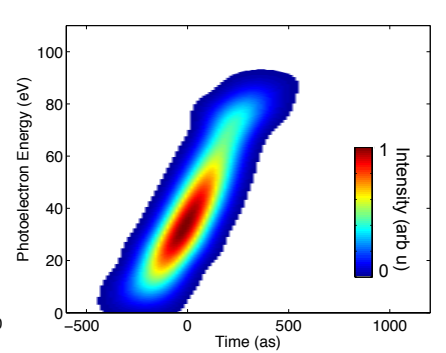

(c)

Figure S7: The retrieved spectral phase (a) and the calculated GDD (b) from PCGPA (blue) and PROOF (green). The retrieved phase from PROOF agrees well with the PCGPA results over the largest amplitude portion of the spectrum, giving an average value of approximately $4500 \mathrm{as}^{2}$. (c) The short time Fourier transform of the retrieved pulse from PROOF, where the slope agrees well with both simulations and PCGPA.

\section{References and Notes}

1. Feng X. et al. Calibration of electron spectrometer resolution in attosecond streak camera. Opt. Express 181316 (2010).

2. Vincenti H. \& Quéré F. Attosecond lighthouse: how to use spatiotemporally coupled light fields to generate isolated attosecond pulses. Phys. Rev. Lett. 108, 113904 (2012).

3. Lewenstein M., Balcou Ph., Ivanov M. Yu., L'Huillier A., \& Corkum P. B. Theory of high-harmonic generation by low-frequency laser fields. Phys. Rev. A 492117 (1994).

4. Yudin G. L. \& Ivanov M. Yu. Nonadiabatic tunnel ionization: looking inside a laser cycle. Phys. Rev. A 64013409 (2001).

5. Hernández-García C. et al. High-order harmonic propagation in gases within the discrete dipole appoximation. Phys. Rev. A 82033432 (2010).

6. López-Martens R. et al. Amplitude and phase control of attosecond light pulses. Phys. Rev. Lett. 94033001 (2005). 
7. Mairesse Y. et al. Attosecond synchronization of high-harmonic soft X-rays. Science 3021540 (2003).

8. Gagnon J., Goulielmakis E. \& Yakovlev V. S. The accurate FROG characterization of attosecond pulses from streaking measurements. Appl. Phys. B 9225 (2008).

9. Chini M., Gilbertson S., Khan S.D. \& Chang Z. Characterizing ultrabroadband attosecond lasers. Opt. Express 1813006 (2010).

10. Zhao K. et al. Tailoring a 67 attosecond pulse through advantageous phase-mismatch. Opt. Lett. 373891 (2012). 